\title{
Comparative efficacy of biological agents in meth- otrexate-refractory rheumatoid arthritis patients: a Bayesian mixed treatment comparison
}

Miyoung Choi ${ }^{1}$, Min Kyung Hyun ${ }^{2}$, Seongmi Choi ${ }^{3}$, Ha Jin Tchoe ${ }^{1}$, Sung Yeon Lee ${ }^{4}$, Kyeong Min Son ${ }^{5}$, Min-Jeong Kim ${ }^{1}$, Young Ok Jung ${ }^{6}$, and Hyun Ah Kim ${ }^{4}$

\begin{abstract}
${ }^{1}$ National Evidence-Based Healthcare Collaboration Agency (NECA), Seoul; ${ }^{2}$ Department of Preventive Medicine, Dongguk University College of Korean Medicine, Seoul; ${ }^{3}$ Korea Appraisal Board, Real Estate R\&D Institute, Seoul; ${ }^{4}$ Department of Internal Medicine, Hallym University Sacred Heart Hospital, Anyang; ${ }^{5}$ Department of Internal Medicine, Hallym University Chuncheon Sacred Heart Hospital, Chuncheon; ${ }^{6}$ Department of Internal Medicine, Hallym University Kangnam Sacred Heart Hospital, Seoul, Korea
\end{abstract}

Received: May 13, 2015

Revised : June 6, 2015

Accepted: June 26, 2015

\section{Correspondence to}

Young Ok Jung, M.D.

Department of Internal Medicine, Hallym University Kangnam Sacred Heart Hospital, 1 Singil-ro, Yeongdeungpo-gu, Seoul o7441, Korea

Tel: +82-2-845-5305

Fax: +82-2-846-4669

E-mail: yjung@hallym.ac.kr
Background/Aims: Biological agents (biologics) targeting proinflammatory signaling have emerged as an important treatment option in rheumatoid arthritis (RA). Despite the clinical effectiveness of biologics for patients with RA who do not respond to 'traditional' disease-modifying anti-rheumatic drugs (DMARDs), there are concerns regarding their cost and long-term safety. In this study, we aimed to compare the efficacy of various biologics and traditional DMARDs in RA patients refractory to methotrexate (MTX).

Methods: Four DMARDs (hydroxychloroquine, sulfasalazine, MTX, leflunomide) and five anti-tumor necrosis factor drugs (adalimumab, etanercept, golimumab, infliximab, and certolizumab) were selected. A systematic search of published studies was performed from inception through July 2013. Randomized trials of adults with MTX-refractory RA comparing two or more of the selected medications were included. Among 7,938 titles identified, in total, 16 head-tohead trials were selected. Two reviewers independently abstracted the study data and assessed methodological quality using the Cochrane Risk of Bias. Comparative efficacy was analyzed using a Bayesian mixed treatment comparison (MTC). Results: In total, 9, 4, and 11 studies were included for the outcome measures of the Health Assessment Questionnaire (HAQ), Disease Activity Score 28-erythrocyte sedimentation rate (DAS28-ESR) < 2.6 (remission), and American College of Rheumatology (ACR) 70 response, respectively. The treatments with the highest efficacy for each outcome measure were certolizumab combined with MTX, golimumab combined with MTX, and certolizumab combined with MTX, respectively.

Conclusions: Based on MTC analysis, using data from published randomized controlled trials, certolizumab and golimumab combined with MTX showed the highest efficacy in the three outcome measures (HAQ, DAS28-ESR < 2.6, and ACR 70 response) in MTX-refractory RA patients.

Keywords: Arthritis, rheumatoid; Antirheumatic agents; Biological products; Mixed treatment comparison 


\section{INTRODUCTION}

Traditional disease-modifying anti-rheumatic drugs (DMARDs), including methotrexate (MTX), sulfasalazine, and leflunomide, have been the cornerstone of the treatment of rheumatoid arthritis (RA). Recently biological agents (biologics), especially tumor necrosis factor antagonists (anti-TNFs, TNF-i), have demonstrated considerable efficacy in treating patients with RA who do not respond or show intolerance to traditional DMARDs [1-3]. Many randomized controlled trials (RCTs) comparing their efficacy with placebo treatment in DMARD-refractory patients have been published, as well as systematic reviews (SRs) and overviews of SRs of these RCTs [4]. Because of their high costs and long-term adverse effects, however, biologics have been under strict regulatory control in many countries, including Korea. One study showed that the majority of RA patients satisfying the American College of Rheumatology (ACR), British Society for Rheumatology, and Japan College of Rheumatology guidelines for use of the TNF- $\alpha$ blockers did not meet the Korean National Health Insurance reimbursement criteria [5]. Because the cost of biologics is much higher than traditional DMARDs, discretion in their use is warranted even in patients refractory to MTX.

Although some DMARD combinations, such as leflunomide or sulfasalazine with MTX, have been shown to be effective in RA patients not responding to MTX monotherapy [5], few studies have compared biologics and combination DMARDs head-to-head in patients refractory to MTX. In the absence of head-to-head trials with relevant comparators, it is possible to compare multiple treatment groups simultaneously by using all the outcomes from direct and indirect comparisons [6]. Mixed treatment comparison (MTC) is one methodology for indirect comparisons, requiring at least one 'closed loop' between non-head-to-head trials and trials should be connected by a common control group (usually, placebo). The similarity and comparability of study designs and other elements should be considered for appropriate use of MTC. However, studies that have applied the MTC methodology to compare DMARDs or biologics in RA patients are few because of the diversity of medications and target outcomes. Thus, the purpose of this study was to find the most effective treatment, including biologics and DMARDs, for MTX-refractory RA patients using the MTC methodology.

\section{METHODS}

\section{Selection of medications}

An expert group consisting of rheumatologists, pharmacists, and evidence-based healthcare methodologists reviewed a list of medications including DMARDs and biologics. They considered feasibility, prescription frequency, and value for patients. Finally, four DMARDs (hydroxychloroquine, sulfasalazine, MTX, leflunomide) and five anti-TNF drugs (adalimumab, etanercept, golimumab, infliximab, and certolizumab) were selected according to the expert group consensus.

\section{Search strategy}

The literature databases searched included four domestic databases, KoreaMed, Korean Medicine Database (KMBASE), Korean Studies Information Service System (KISS), and Korean Institute of Science and Technology Information (KISTI), and the core databases MEDLINE, EMBASE, and the Cochrane Library.

Studies that met the following criteria were included: (1) subjects were RA patients not responsive to MTX, (2) treatment consisted of one of the nine medications selected for evaluation, (3) RCT study design, and (4) reported patient outcomes included ACR responders 20/50/70, the Health Assessment Questionnaire (HAQ), or the Disease Activity Score 28-erythrocyte sedimentation rate (DAS28-ESR).

Studies of animal trials or preclinical studies and non-original articles, such as review articles, editorials, letter, and comments, were excluded. Articles not published in Korean or English and studies with duplicate subjects (studies using the same outcome indicators published in duplicate) were also excluded.

Two reviewers screened all titles and abstracts identified by the searches independently. Full manuscripts of studies screened as potentially relevant by either reviewer were obtained and assessed by the two reviewers independently. Discrepancies were resolved by consensus.

\section{Data extraction and quality assessment}

The two reviewers extracted data from each study in- 
dependently. The data were reviewed for consistency between the two extractors, and disagreements were resolved by consensus. For each study, data extraction details included design, selection criteria, population characteristics, treatments, outcome measures, length of follow-up, and results.

\section{Major outcomes}

(1) The HAQ survey has eight sections on how the treatment improves daily activities, such as dressing, arising, eating, walking, hygiene, reaching, gripping, and activities.

(2) The ACR responder rate is the rate of patients with a 20\%/50\%/70\% improvement in tender and swollen joint counts and improvement in three of the five remaining ACR-core set measures: patient and physician global assessments, pain, disability, and an acute phase reactant.

(3) DAS28 is a combined index using tender/swollen joint count, ESR, and a general health assessment on a visual analog scale.

Two reviewers independently assessed the methodological quality of the included studies using the Cochrane Risk of Bias (RoB). The results of the RoB evaluation were converted to graphical displays using the RevMan 5.2 program (The Nordic Cochrane Center, Copenhagen, Denmark).

\section{Statistical analysis}

Bayesian MTC was carried out by designating the control group as placebo combined with MTX due to lack of clinical trials with direct comparisons. A random effects model was applied because of the heterogeneity between trials. Continuous variables, such as mean change from baseline, median range, and standard deviation, were converted according to the formula in the Cochrane Handbook [7].

Comparative efficacy was analyzed using a MTC. In a Bayesian meta-analysis, inference on a parameter of interest is made based on the posterior distribution obtained from a prior distribution combined with the likelihood function. The 'posterior probability distribution' allows calculating the probability of the case in which the competing intervention is best. In addition, a pooled estimate with a credible interval (CrI), referring to the probability of including a parameter, is obtained using a posterior probability distribution and the hypothesis of interest is tested [6]. If the CrI does not include ' 1 ' and if the probability is close to 1 , then the treatment could be interpreted as effective. The 'best' is the relative estimate effect for all treatments in a comparison ranking. The highest effective treatment was decided based on considering the 'best' and the 'posterior probability distribution.' Consistency between evidence sources was assessed by comparing models with and without inconsistency factors. The software used for statistical analyses was WinBUGS 14 (MRC Biostatistics Unit, Cambridge, UK).

\section{RESULTS}

\section{Selected studies}

Of the 7,938 citations identified through our literature search, 7,789 were excluded through exclusion of duplicates and screening of titles and abstracts. In addition, an overview of SRs was performed up to May 2013 according to a previously published methodology [8]. Among 52 SRs, we selected two high-quality SRs, one each for biologics [9] and DMARDs [10]. Three more SRs including trials with DMARDs were added $[5,11,12]$. All randomized trials obtained from SRs were pooled and updated from January 2009 to July 2013 (Table 1, Fig. 1) [13-28].

The mean age of patients included in the trials was 52.9 years and the mean rheumatoid factor-positive rate was $76.6 \%$. RA treatment duration was from 4 to 13.1 years. The follow-up period was from 1.6 months to 1 year. Characteristics of each study are shown in Table 1.

\section{Risk of bias}

The quality of the 16 selected randomized trials was assessed using the Cochrane RoB tool (Fig. 2). Generally, studies without the phrase 'random assignment' and without a specific method for random number generation and assignment number-concealing measures were considered to be 'uncertain.' There were many studies in which the risk of selection bias was perceived as 'uncertain.' However, most studies had a low risk in terms of attrition or reporting bias. 


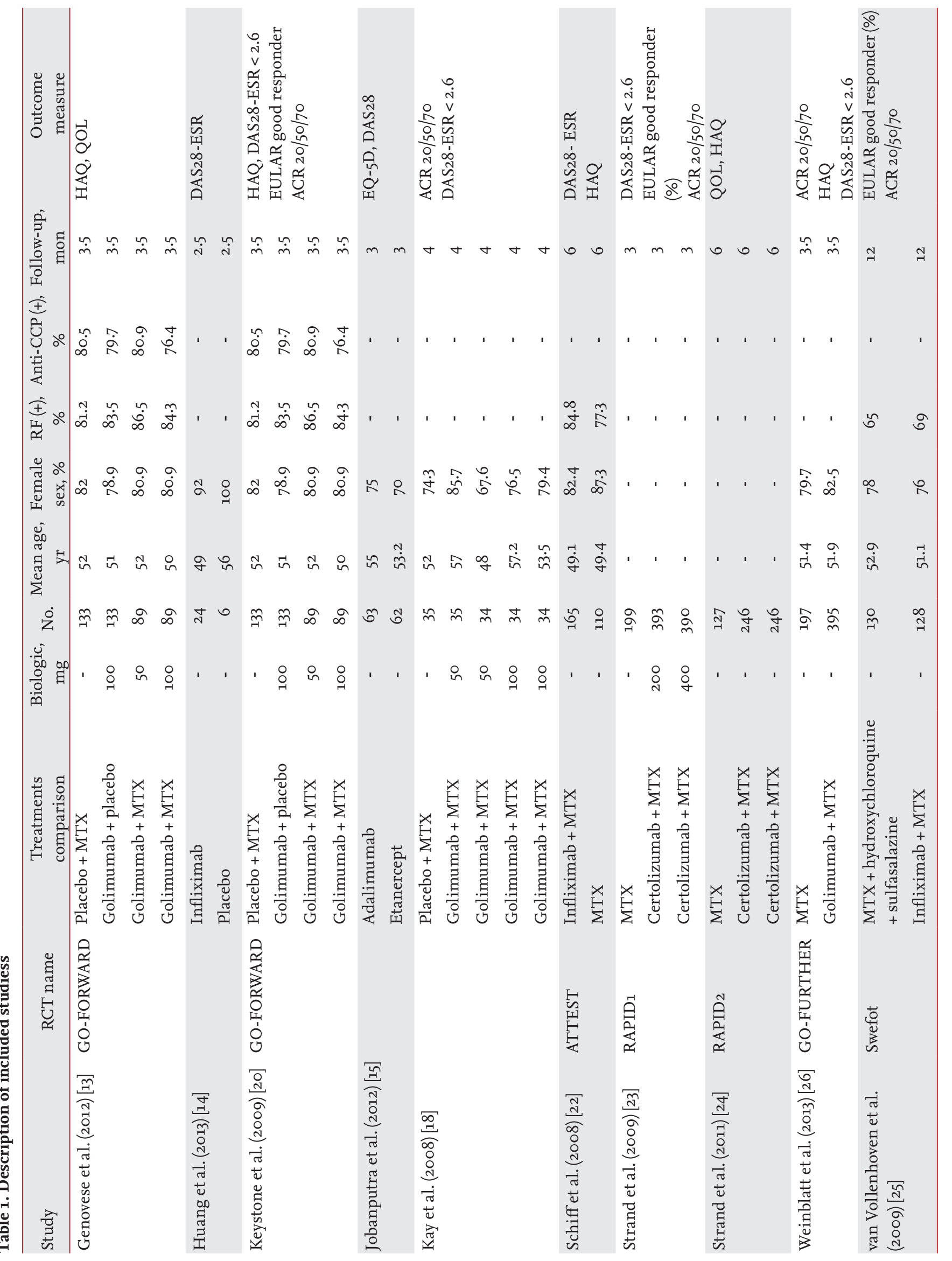




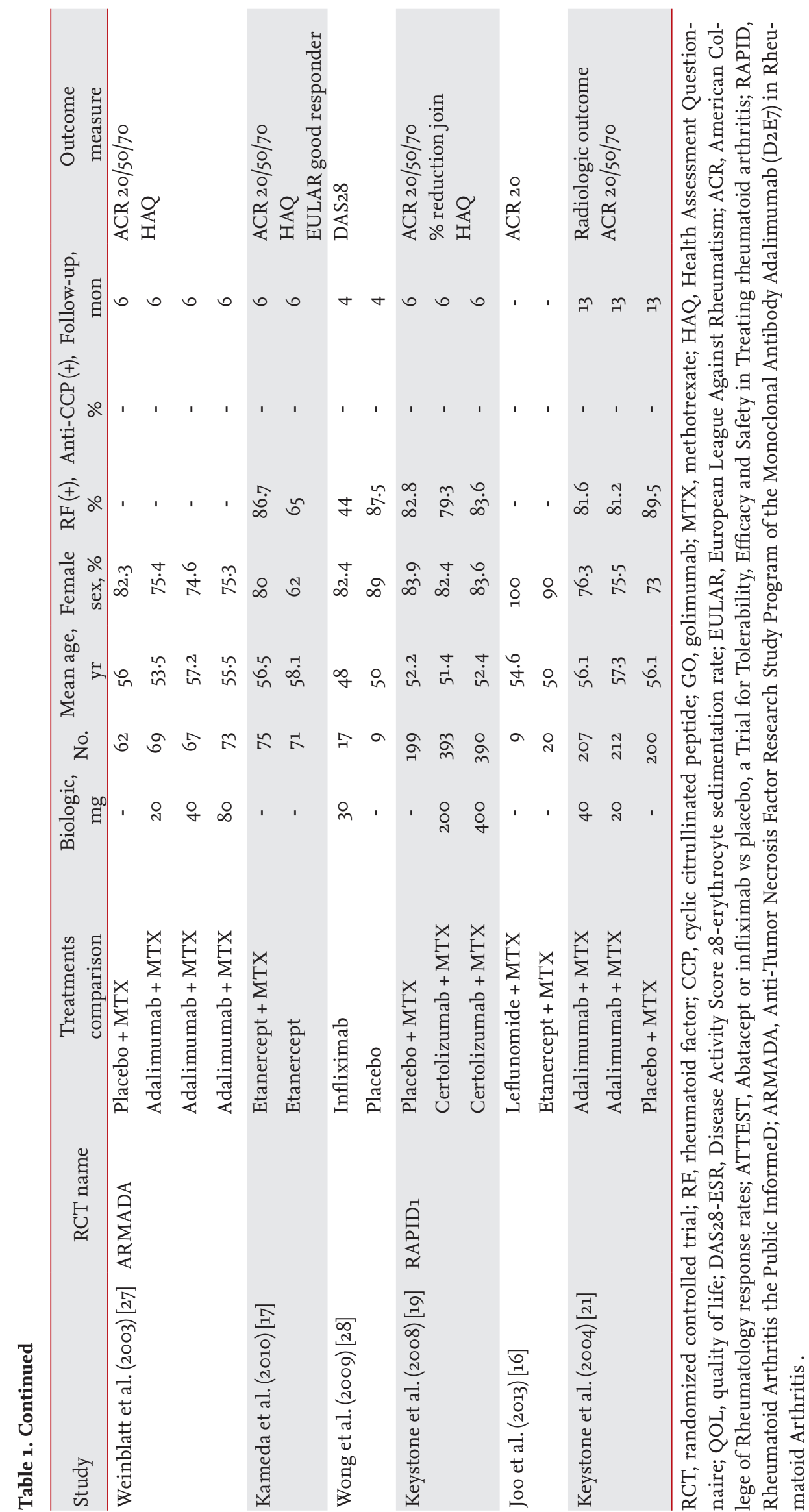




\section{Comparative efficacy of treatments based on the Health Assessment Questionnaire}

In total, nine studies were included in the HAQ analysis (Fig. 3). The treatment that showed the highest efficacy in improving HAQ scores (with the highest mean difference and probability of distribution) was the combination of certolizumab with MTX, followed by the combinations of golimumab with MTX and adalimumab with MTX (Table 2). The effect size for each treatment was similar in direction to the mean difference, with the highest value for certolizumab with MTX (Table 3). No study included in this analysis had a traditional DMARD arm for comparison.

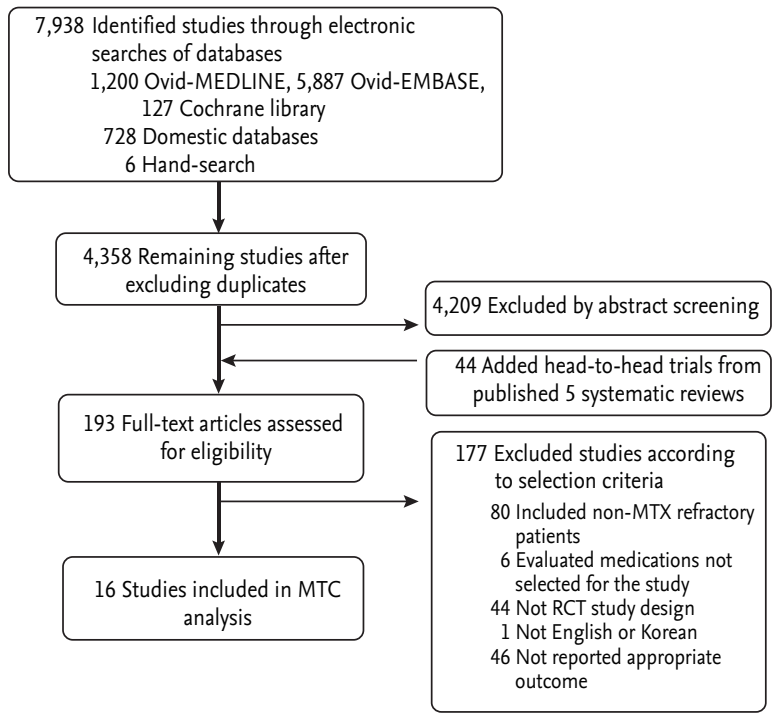

Figure 1. Flow diagram of study selection. MTX, methotrexate; MTC, mixed treatment comparison.

\section{Comparative efficacy of treatments based on DAS28 $<2.6$ (remission)}

In total, four studies were included in the DAS28-ESR < 2.6 (remission) analysis (Fig. 3). The most effective treatment for DAS28-ESR < 2.6 (remission), with the highest odds ratio (OR) and probability OR, compared with MTX, was golimumab with MTX (OR, 24.500; 95\% CrI, 3.508 to 99.520) (Table 4). The effect size was highest for golimumab with MTX (Table 5). No study included in this analysis had a traditional DMARD arm for comparison.

\section{Comparative efficacy of treatments based on ACR response}

In total, 11 studies were included for the ACR 70 response analysis (Fig. 3). The most effective treatment based on ACR 70 response was certolizumab with MTX (OR, 10.460; 95\% CrI, 3.660 to 24.410) (Table 6); this combination also had the highest effect size (Table 7). ACR 20 and ACR 50 response rates could not be analyzed because of an insufficient number of studies. Combi-

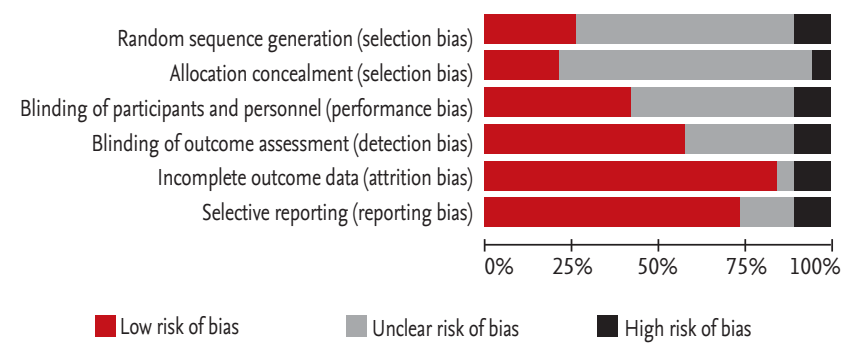

Figure 2. Risk of bias assessment summary.

Table 2. Comparative effectiveness on Health Assessment Questionnaire scores

\begin{tabular}{|c|c|c|c|c|c|}
\hline Treatment & $\begin{array}{c}\text { No. of } \\
\text { studies }\end{array}$ & $\begin{array}{c}\text { No. of } \\
\text { participants }\end{array}$ & $\begin{array}{c}\text { Mean difference } \\
\text { (95\% CrI) }\end{array}$ & Best $^{\mathrm{a}}$ & $\begin{array}{l}\text { Prob.d }{ }^{b} \\
\text { (mean) }\end{array}$ \\
\hline MTX (placebo + MTX) & 8 & 1,004 & - & 0.001 & - \\
\hline CERT + MTX & 1 & 246 & $-0.400(-0.945$ to 0.131$)$ & 0.2347 & 0.9572 \\
\hline $\mathrm{GOL}+\mathrm{MTX}$ & 2 & 200 & $-0.125(-0.571$ to 0.379$)$ & 0.0382 & 0.7256 \\
\hline ADAL + MTX & 2 & 274 & $-0.354(-1.557$ to 0.839$)$ & 0.3072 & 0.7191 \\
\hline ADAL & 1 & 67 & $-0.341(-2.053$ to 1.363$)$ & 0.3778 & 0.6530 \\
\hline GOL & 2 & 200 & $-0.082(-0.689$ to 0.526$)$ & 0.0413 & 0.6220 \\
\hline
\end{tabular}

CrI, credible interval (the probability to include a parameter); Prob.d, probability distribution; MTX, methotrexate; CERT, certolizumab; GOL, golimumab; ADAL, adalimumab.

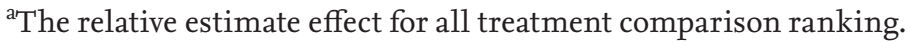

${ }^{\mathrm{b}}$ Statistical significance: probability distribution > 0.9. 
Table 3. Effect sizes for Health Assessment Questionnaire

\begin{tabular}{lccccc}
\hline \multirow{2}{*}{ Comparator } & \multicolumn{5}{c}{ Effect size by treatment, mean difference (95\% CI) } \\
\cline { 2 - 6 } & ADAL & ADAL + MTX & CERT + MTX & GOL & GOL + MTX \\
\hline Placebo + MTX & $-0.35(-0.56$ to -0.14$)$ & $-0.34(-0.44$ to -0.25$)$ & $-0.40(-0.41$ to -0.39$)$ & $-0.12(-0.16$ to -0.08$)$ & $-0.09(-0.12$ to -0.06$)$ \\
ADAL & - & $0.01(-0.22$ to 0.24$)$ & $-0.05(-0.26$ to 0.16$)$ & $0.23(0.16$ to 0.44$)$ & $0.26(0.48$ to 0.47$)$ \\
ADAL + MTX & - & - & $-0.06(-0.16$ to 0.04$)$ & $0.22(0.12$ to 0.32$)$ & $0.25(0.15$ to 0.35$)$ \\
CERT + MTX & - & - & - & $0.28(0.08$ to 0.48$)$ & $0.31(0.28$ to 0.34$)$ \\
GOL & - & - & - & - & $0.2(-0.02$ to 0.08$)$ \\
\hline
\end{tabular}

CI, confidence interval; ADAL, adalimumab; MTX, methotrexate; CERT, certolizumab; GOL, golimumab.
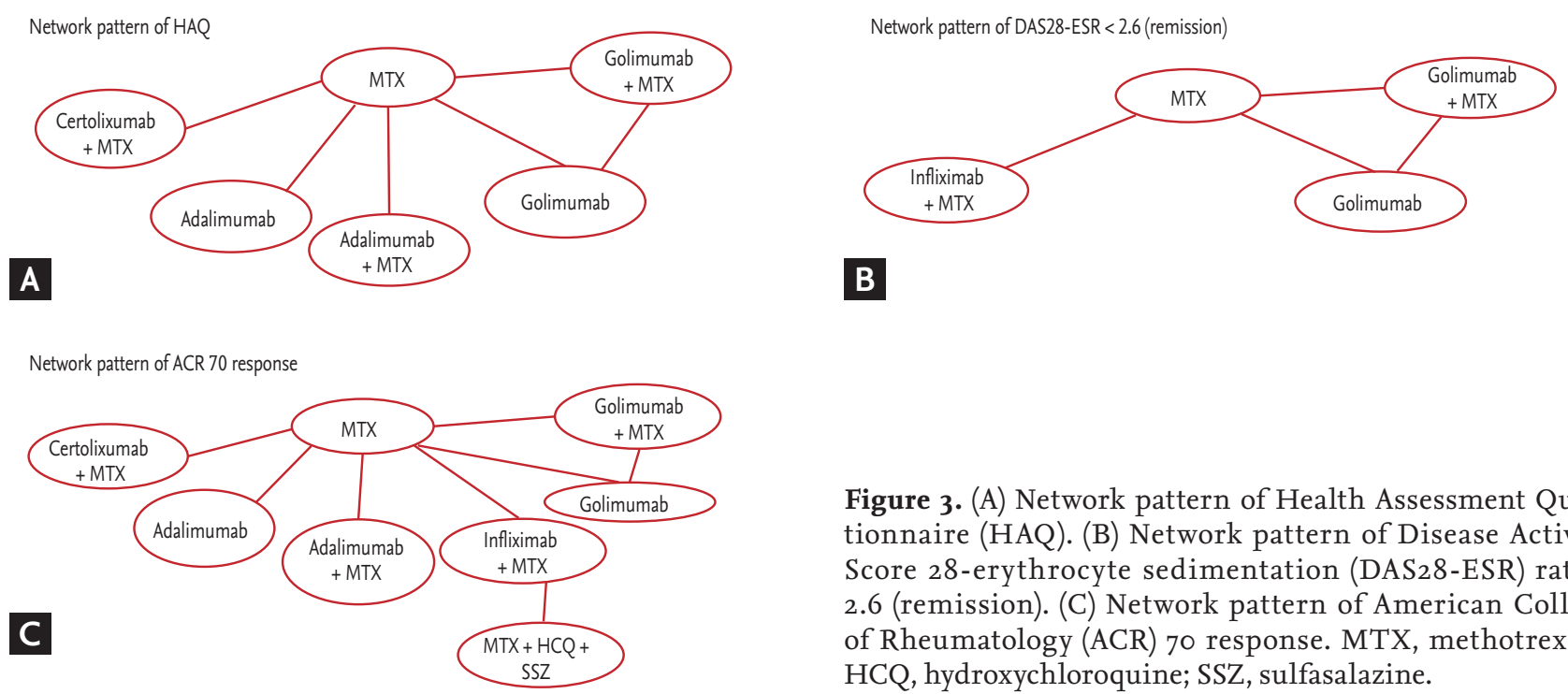

Figure 3. (A) Network pattern of Health Assessment Questionnaire (HAQ). (B) Network pattern of Disease Activity Score 28-erythrocyte sedimentation (DAS28-ESR) rate < 2.6 (remission). (C) Network pattern of American College of Rheumatology (ACR) 70 response. MTX, methotrexate; HCQ, hydroxychloroquine; SSZ, sulfasalazine.

Table 4. Comparative effectiveness on Disease Activity Score 28 -erythrocyte sedimentation rate $<2.6$ (remission)

\begin{tabular}{|c|c|c|c|c|c|}
\hline Treatment & $\begin{array}{l}\text { No. of } \\
\text { studies }\end{array}$ & $\begin{array}{c}\text { No. of } \\
\text { participants }\end{array}$ & OR $(95 \% \mathrm{CrI})$ & Best $^{\mathrm{a}}$ & $\begin{array}{c}\text { Probability OR } \\
\text { (mean) }\end{array}$ \\
\hline MTX (placebo + MTX) & 4 & 368 & - & 0.00003 & - \\
\hline $\mathrm{GOL}+\mathrm{MTX}$ & 3 & 213 & $24.500(3.508-99.520)$ & 0.6545 & 0.9989 \\
\hline GOL & 1 & 133 & $18.830(0.625-93.290)$ & 0.1606 & 0.9537 \\
\hline IFX + MTX & 1 & 165 & $15.160(0.552-73.820)$ & 0.1849 & 0.9459 \\
\hline
\end{tabular}

OR, odds ratio; CrI, credible interval (the probability to include a parameter); MTX, methotrexate; GOL, golimumab; IFX, infliximab.

${ }^{\text {a }}$ The relative estimate effect for all treatment comparison ranking.

nation DMARDs with MTX, hydroxychloroquine, and sulfasalazine had the lowest probability OR among the treatments compared.

\section{DISCUSSION}

In this study, we sought to compare the efficacy of vari- ous biologics and traditional DMARDs for MTX-refractory RA patients using a MTC methodology. Unfortunately, the number of traditional DMARD studies was not sufficient to allow an appropriate MTC analysis. The results showed that the combinations of golimumab with MTX and certolizumab with MTX were the most effective treatments in terms of $\mathrm{DAS}_{2} 8$ remission and ACR 70 responses for RA patients who did not respond 
Table 5. Effect sizes for Disease Activity Score 28-erythrocyte sedimentation rate $<2.6$ (remission)

\begin{tabular}{lccc}
\hline \multirow{2}{*}{ Comparator } & \multicolumn{3}{c}{ Effect size by treatment, OR (95\% CI) } \\
\cline { 2 - 4 } & GOL & GOL + MTX & IFX + MTX \\
\hline Placebo + MTX & $5.91(1.28-27.18)$ & $14.40(5.34-38.79)$ & $5.20(1.51-17.89)$ \\
GOL & - & $2.44(0.39-15.06)$ & $0.88(0.12-6.28)$ \\
GOL + MTX & - & - & $0.36(0.07-1.77)$ \\
\hline
\end{tabular}

OR, odds ratio; CI, confidence interval; GOL, golimumab; MTX, methotrexate; IFX, infliximab.

Table 6. Comparative effectiveness on American College of Rheumatology 70 response

\begin{tabular}{lccccc}
\hline Treatment & $\begin{array}{c}\text { No. of } \\
\text { studies }\end{array}$ & $\begin{array}{c}\text { No. of } \\
\text { participants }\end{array}$ & OR (95\% CrI) & $\begin{array}{c}\text { Best } \\
\text { Probability OR } \\
(\mathrm{mean})\end{array}$ \\
\hline MTX (placebo + MTX) & 10 & 1,289 & - & 0 & - \\
CERT + MTX & 2 & 786 & $10.460(3.660-24.410)$ & 0.3671 & 0.9990 \\
ADAL & 1 & 67 & $13.300(1.708-52.970)$ & 0.3602 & 0.9922 \\
ADAL + MTX & 2 & 274 & $8.086(2.762-19.650)$ & 0.1599 & 0.9985 \\
GOL & 1 & 133 & $3.422(0.624-10.370)$ & 0.0131 & 0.9276 \\
GOL + MTX & 4 & 608 & $6.917(3.137-15.250)$ & 0.0703 & 0.9997 \\
IFX + MTX & 2 & 293 & $4.084(0.939-11.670)$ & 0.0153 & 0.9713 \\
MTX + HCQ + SSZ & 1 & 130 & $3.457(0.283-10.940)$ & 0.0141 & 0.7695 \\
\hline
\end{tabular}

OR, odds ratio; CrI, credible interval (the probability to include a parameter); MTX, methotrexate; CERT, certolizumab; ADAL, adalimumab; GOL, golimumab; IFX, infliximab; HCQ, hydroxychloroquine; SSZ, sulfasalazine.

Table 7. Effect sizes for American College of Rheumatology 70

\begin{tabular}{lcccccc}
\hline \multirow{2}{*}{ Comparator } & \multicolumn{5}{c}{ Effect size by treatment, OR (95\% CI) } \\
\cline { 2 - 7 } & ADAL & ADAL + MTX & CERT + MTX & GOL & GOL + MTX & IFX + MTX \\
\hline Placebo + MTX & $7.22(2.01-25.97)$ & $6.61(3.48-12.56)$ & $8.74(3.75-20.41)$ & $2.08(0.69-6.26)$ & $5.69(3.47-9.33)$ & $3.10(1.47-6.50)$ \\
ADAL & - & $0.92(0.22-3.83)$ & $1.21(0.26-5.62)$ & $0.29(0.53-1.56)$ & $0.79(0.2-3.11)$ & $0.43(0.10-1.89)$ \\
ADAL + MTX & - & - & $1.32(0.46-0.83)$ & $0.32(0.14-0.71)$ & $0.86(0.38-1.94)$ & $0.47(0.18-1.25)$ \\
CERT + MTX & - & - & - & $0.24(0.06-0.97)$ & $0.65(0.24-1.74)$ & $0.36(0.12-1.10)$ \\
GOL & - & - & - & - & $2.74(0.82-9.16)$ & $1.49(0.39-5.63)$ \\
GOL + MTX & - & - & - & - & - & $0.55(0.22-1.33)$ \\
\hline
\end{tabular}

OR, odds ratio; CI, confidence interval; ADAL, adalimumab; MTX, methotrexate; CERT, certolizumab; GOL, golimumab; IFX, infliximab.

to MTX treatment alone. For traditional DMARDs, only one trial was available for analysis of ACR 70 response, and the result showed that MTX combined with sulfasalazine and hydroxychloroquine was substantially inferior to biologics combinations (Table 6).

MTC analysis enables a comparison of the efficacy of biologic and DMARD treatment for MTX-refractory patients, even in the absence of direct comparisons. Indirect comparisons were unavoidable, because head- to-head trials for the medications of interest are rare, and often, biologics were compared with placebo, not to DMARDs or even other biologics [29]. While a 'traditional' meta-analysis summarizes the results of trials that have evaluated the same treatment/placebo combination, an MTC analysis consists of a network of treatment effects for all possible pairwise comparisons from RCTs, including both direct and indirect comparisons. A common comparator should exist, on which the treat- 
ment effects compared are anchored [30].

Previously, many studies have reported direct comparisons of a biological agent combined with MTX and MTX monotherapy in MTX-refractory patients, which came to the conclusion that biologics are significantly more effective in these patients [31]. However, studies reporting the results of indirect comparisons of the efficacy of different biologics in RA found no statistically significant difference between them [2,32]. Certolizumab pegol was found superior to infliximab in some studies [33], which is partly in line with our results, showing that certolizumab combined with MTX improved both HAQ scores and ACR 70 most effectively. Certolizum$\mathrm{ab}$ pegol consists of a humanized Fab fragment fused to a polyethylene glycol (PEG) moiety. The addition of the PEG structure increases the half-life up to 14 days and facilitates distribution into inflamed tissues in animal models. This different structure may be the basis of the additional effectiveness [34,35]. However, the RAPID trials, which were included in our analysis, withdrew non-responsive patients at weeks 12 and 14, and accordingly showed a high early withdrawal rate in the placebo group, which could have resulted in the high OR rate of certolizumab pegol compared with the placebo [31]. Recent meta-analysis updates of the comparative safety of TNF inhibitors suggest a higher risk of serious infection associated with certolizumab pegol, as well as adalimumab and infliximab, guiding clinical decision-making in balancing efficacy and safety in the management of RA.

At the time of preparation of this manuscript, randomized trials directly comparing the combination of a biologic agent plus MTX with a combination of traditional DMARDs [36] had been published, showing no difference between the two treatments. The combination of traditional DMARDs versus biologics has rarely been reported in systemic reviews, despite the huge implications concerning the cost differences between treatments with and without biologics. A recent meta-analysis of 38 RCTs showed that combination treatment with a biologic agent (a TNF-i or abatacept) and a DMARD was not superior to two or three DMARDs in combination, including or excluding low-dose glucocorticoids, in preventing structural joint damage [30].

While the effectiveness of biologics in RA patients unresponsive to MTX has been settled in many clinical tri- als and meta-analyses thereof, therapeutic equivalence and noninferiority continue to be debated. In an equivalence analysis of seven RCTs, MTX plus adalimumab or certolizumab led to superior ACR 50 responses in comparison with MTX monotherapy, but not for MTX with etanercept or golimumab [37]. Head-to-head indirect comparisons between individual biologics showed no significant difference, but failed to demonstrate equivalence. The high degree of clinical heterogeneity in the included trials and short duration of follow-up, compromising external validity, were considered the main reasons for the failure to demonstrate equivalence.

Our study has several limitations. Although we included only RCTs, the MTC analysis still showed statistical heterogeneity and inconsistency. Indirect comparison requires similarity, homogeneity of trials, and consistency of evidence [38]. Study procedures, such as blinding and allocation concealment, may affect the heterogeneity $[6,39]$. Additionally, differences in disease duration and medication dose between trials might affect trial homogeneity. A second limitation of this study was publication bias, because only published studies were included. Although a comprehensive search was done, the final RCT studies included in the MTC analysis for comparison were more numerous for trials testing biologics than traditional DMARDs. Because of the timeframe, most recent studies comparing biologics and traditional DMARDs were not included.

In conclusion, based on an MTC analysis using data from published RCTs, the combinations of certolizum$\mathrm{ab}$ or golimumab with MTX are most effective in improving HAQ, DAS28-ESR < 2.6, and ACR 70 response in MTX-refractory RA patients.

\section{KEY MESSAGE}

1. Mixed treatment comparison (MTC) analysis enables a comparison of efficacy of biologics and traditional disease-modifying anti-rheumatic drugs (DMARDs) treatment in the absence of direct comparison for methotrexate (MTX)-refactory rheumatoid arthritis (RA) patients.

2. The combination of cetolizumab or golimumab with MTX were the most effective treatment in MTX-refractory RA patients based on MTC analysis. 
3. The number of traditional DMARDs studies was not sufficient to allow an appropriate MTC analysis.

\section{Conflict of interest}

No potential conflict of interest relevant to this article was reported.

\section{Acknowledgments}

This study was funded by the National Evidence-based Healthcare Collaborating Agency (NECA), project no. $\mathrm{NC}-2013-012$, and by a grant (A120960) from the Korean Health Technology R\&D Project, Ministry of Health and Welfare, Republic of Korea.

\section{REFERENCES}

1. Schmitz S, Adams R, Walsh CD, Barry M, FitzGerald O. A mixed treatment comparison of the efficacy of anti-TNF agents in rheumatoid arthritis for methotrexate non-responders demonstrates differences between treatments: a Bayesian approach. Ann Rheum Dis 2012;71:225-230.

2. Devine EB, Alfonso-Cristancho R, Sullivan SD. Effectiveness of biologic therapies for rheumatoid arthritis: an indirect comparisons approach. Pharmacotherapy 2011;31:39-51.

3. Gallego-Galisteo M, Villa-Rubio A, Alegre-del Rey E, Marquez-Fernandez E, Ramos-Baez JJ. Indirect comparison of biological treatments in refractory rheumatoid arthritis. J Clin Pharm Ther 2012;37:301-307.

4. Singh JA, Cameron DR. Summary of AHRQ's comparative effectiveness review of drug therapy for rheumatoid arthritis (RA) in adults: an update. J Manag Care Pharm 2012;18(4 Supp C):S1-S18.

5. Golicki D, Newada M, Lis J, Pol K, Hermanowski T, Tlustochowicz M. Leflunomide in monotherapy of rheumatoid arthritis: meta-analysis of randomized trials. Pol Arch Med Wewn 2012;122:22-32.

6. Ahn J, Choi S, Cho S, Tchoe HJ. Bayesian Meta-Analysis Method. Seoul: National Evidence-based Healthcare Collaborating Agency, 2014.

7. Higgins JP, Green S. Cochrane Handbook for Systematic Reviews of Interventions. Chichester: Wiley-Blackwell, 2008.
8. Whitlock EP, Lin JS, Chou R, Shekelle P, Robinson KA. Using existing systematic reviews in complex systematic reviews. Ann Intern Med 2008;148:776-782.

9. Aaltonen KJ, Virkki LM, Malmivaara A, Konttinen YT, Nordstrom DC, Blom M. Systematic review and meta-analysis of the efficacy and safety of existing TNF blocking agents in treatment of rheumatoid arthritis. PLoS One 2012;7:e30275.

10. Gaujoux-Viala C, Smolen JS, Landewe R, et al. Current evidence for the management of rheumatoid arthritis with synthetic disease-modifying antirheumatic drugs: a systematic literature review informing the EULAR recommendations for the management of rheumatoid arthritis. Ann Rheum Dis 2010;69:1004-1009.

11. Turkstra E, Ng SK, Scuffham PA. A mixed treatment comparison of the short-term efficacy of biologic disease modifying anti-rheumatic drugs in established rheumatoid arthritis. Curr Med Res Opin 2011;27:1885-1897.

12. Graudal N, Jurgens G. Similar effects of disease-modifying antirheumatic drugs, glucocorticoids, and biologic agents on radiographic progression in rheumatoid arthritis: meta-analysis of 70 randomized placebo-controlled or drug-controlled studies, including 112 comparisons. Arthritis Rheum 2010;62:2852-2863.

13. Genovese MC, Han C, Keystone EC, et al. Effect of golimumab on patient-reported outcomes in rheumatoid arthritis: results from the GO-FORWARD study. J Rheumatol 2012;39:1185-1191.

14. Huang J, Xie B, Li Q, et al. Infliximab reduces CD147, MMP-3, and MMP-9 expression in peripheral blood monocytes in patients with active rheumatoid arthritis. Eur J Pharmacol 2013;698:429-434.

15. Jobanputra P, Maggs F, Deeming A, et al. A randomised efficacy and discontinuation study of etanercept versus adalimumab (RED SEA) for rheumatoid arthritis: a pragmatic, unblinded, non-inferiority study of first TNF inhibitor use: outcomes over 2 years. BMJ Open 2012;2:e001395.

16. Joo K, Park W, Kwon SR, Lim MJ. Safety and efficacy evaluation for the addition of either etanercept or leflunomide in Korean rheumatoid arthritis patients inadequately responding to methotrexate. J Rheum Dis 2013;20:166-171.

17. Kameda H, Ueki Y, Saito K, et al. Etanercept (ETN) with methotrexate (MTX) is better than ET'N monotherapy in patients with active rheumatoid arthritis despite MTX 
therapy: a randomized trial. Mod Rheumatol 2010;20:531538.

18. Kay J, Matteson EL, Dasgupta B, et al. Golimumab in patients with active rheumatoid arthritis despite treatment with methotrexate: a randomized, double-blind, placebo-controlled, dose-ranging study. Arthritis Rheum 2008;58:964-975.

19. Keystone E, Van Der Heijde D, Mason D Jr, et al. Certolizumab pegol plus methotrexate is significantly more effective than placebo plus methotrexate in active rheumatoid arthritis: findings of a fifty-two-week, phase III, multicenter, randomized, double-blind, placebo-controlled, parallel-group study. Arthritis Rheum 2008;58:3319-3329.

20. Keystone EC, Genovese MC, Klareskog L, et al. Golimumab, a human antibody to tumour necrosis factor \{alpha\} given by monthly subcutaneous injections, in active rheumatoid arthritis despite methotrexate therapy: the GO-FORWARD Study. Ann Rheum Dis 2009;68:789-796.

21. Keystone EC, Schiff MH, Kremer JM, et al. Once-weekly administration of $50 \mathrm{mg}$ etanercept in patients with active rheumatoid arthritis: results of a multicenter, randomized, double-blind, placebo-controlled trial. Arthritis Rheum 2004;50:353-363.

22. Schiff M, Keiserman M, Codding C, et al. Efficacy and safety of abatacept or infliximab vs placebo in ATTEST: a phase III, multi-centre, randomised, double-blind, placebo-controlled study in patients with rheumatoid arthritis and an inadequate response to methotrexate. Ann Rheum Dis 2008;67:1096-1103.

23. Strand V, Mease P, Burmester GR, et al. Rapid and sustained improvements in health-related quality of life, fatigue, and other patient-reported outcomes in rheumatoid arthritis patients treated with certolizumab pegol plus methotrexate over 1 year: results from the RAPID 1 randomized controlled trial. Arthritis Res Ther 2009;11:R170.

24. Strand V, Smolen JS, van Vollenhoven RF, et al. Certolizumab pegol plus methotrexate provides broad relief from the burden of rheumatoid arthritis: analysis of patient-reported outcomes from the RAPID 2 trial. Ann Rheum Dis 2011;70:996-1002.

25. van Vollenhoven RF, Ernestam S, Geborek P, et al. Addition of infliximab compared with addition of sulfasalazine and hydroxychloroquine to methotrexate in patients with early rheumatoid arthritis (Swefot trial): 1-year re- sults of a randomised trial. Lancet 2009;374:459-466.

26. Weinblatt ME, Bingham CO 3rd, Mendelsohn AM, et al. Intravenous golimumab is effective in patients with active rheumatoid arthritis despite methotrexate therapy with responses as early as week 2: results of the phase 3, randomised, multicentre, double-blind, placebo-controlled GO-FURTHER trial. Ann Rheum Dis 2013;72:381389.

27. Weinblatt ME, Keystone EC, Furst DE, et al. Adalimumab, a fully human anti-tumor necrosis factor alpha monoclonal antibody, for the treatment of rheumatoid arthritis in patients taking concomitant methotrexate: the ARMADA trial. Arthritis Rheum 2003;48:35-45.

28. Wong M, Oakley SP, Young L, et al. Infliximab improves vascular stiffness in patients with rheumatoid arthritis. Ann Rheum Dis 2009;68:1277-1284.

29. Canadian Agency for Drugs and Technologies in Health. Therapeutic review. Clinical and economic overview: biological response modifier agents for adults with rheumatoid arthritis [Internet]. Ottawa (ON): Canadian Agency for Drugs and Technologies in Health, c2010 [cited 2016 Apr 22]. Available from: https://www.cadth.ca/media/pdf/ TR_RA_Clinical_and_Economic_Overview_e.pdf.

30. Graudal N, Hubeck-Graudal T, Tarp S, Christensen R, Jurgens G. Effect of combination therapy on joint destruction in rheumatoid arthritis: a network meta-analysis of randomized controlled trials. PLoS One 2014;9:e106408.

31. Baji P, Pentek M, Czirjak L, et al. Efficacy and safety of infliximab-biosimilar compared to other biological drugs in rheumatoid arthritis: a mixed treatment comparison. Eur J Health Econ 2014;15 Suppl 1:S53-S64.

32. Singh JA, Christensen R, Wells GA, et al. A network meta-analysis of randomized controlled trials of biologics for rheumatoid arthritis: a Cochrane overview. CMAJ 2009;181:787-796.

33. Launois R, Avouac B, Berenbaum F, et al. Comparison of certolizumab pegol with other anticytokine agents for treatment of rheumatoid arthritis: a multiple-treatment Bayesian metaanalysis. J Rheumatol 2011;38:835-845.

34. Nesbitt A, Fossati G, Bergin M, et al. Mechanism of action of certolizumab pegol (CDP870): in vitro comparison with other anti-tumor necrosis factor alpha agents. Inflamm Bowel Dis 2007;13:1323-1332.

35. Nesbitt A, Fossati G, Brown D, Henry A, Palframan R, Stephens S. Effect of structure of conventional anti-TNFs and certolizumab pegol on mode of action in rheuma- 
toid arthritis. Ann Rheum Dis 2007;66(Suppl 2):296.

36. Goekoop-Ruiterman YP, de Vries-Bouwstra JK, Allaart CF, et al. Clinical and radiographic outcomes of four different treatment strategies in patients with early rheumatoid arthritis (the BeSt study): a randomized, controlled trial. Arthritis Rheum 2005;52:3381-3390.

37. Messori A, Fadda V, Maratea D, et al. Biological drugs for the treatment of rheumatoid arthritis by the subcutaneous route: interpreting efficacy data to assess statistical equivalence. Ther Adv Musculoskelet Dis 2014;6:207-216.

38. Jansen JP, Fleurence R, Devine B, et al. Interpreting indirect treatment comparisons and network meta-analysis for health-care decision making: report of the ISPOR Task Force on Indirect Treatment Comparisons Good Research Practices: part 1. Value Health 2011;14:417-428.

39. Ades AE, Madan J, Welton NJ. Indirect and mixed treatment comparisons in arthritis research. Rheumatology (Oxford) 2011;50 Suppl 4:iv5-iv9. 